\title{
Ky Fan minimax inequalities for set-valued mappings
}

Yu Zhang ${ }^{1 *}$ and Sheng-Jie $\mathrm{Li}^{1,2}$

* Correspondence:

zhangyu198606@sina.com

${ }^{1}$ College of Mathematics and

Statistics, Chongqing University, Chongqing 401331, China

Full list of author information is

available at the end of the article

\section{Abstract}

In this article, by virtue of the Kakutani-Fan-Glicksberg fixed point theorem, two types of Ky Fan minimax inequalities for set-valued mappings are obtained. Some examples are given to illustrate our results.

Mathematics Subject Classification (2010): 49J35; 49K35; $90 \mathrm{C} 47$.

Keywords: minimax inequality, fixed point theorem, set-valued mapping

\section{Introduction}

It is well known that Ky Fan minimax inequalities play a very important role in many fields, such as variational inequalities, game theory, mathematical economics, control theory, and fixed point theory. Because of its wide applications, Ky Fan minimax inequalities have been generalized in various ways. Since 1960s, Ky Fan minimax theorems of the real-valued functions have been discussed, such as [1-4] and references therein.

In recent years, based on the development of vector optimization, a great deal of articles have devoted to the study of the Ky Fan minimax theorems for vector-valued functions. In [5], Chen proved a Ky Fan minimax inequality for vector-valued mappings on $H$-spaces by using a generalized Fan's section theorem and a generalized Browder's fixed point theorem. Chang et al. [6] obtained a Ky Fan minimax inequality for vector-valued mappings on $W$-spaces by applying a generalized section theorem and a generalized fixed point theorem. Li and Wang [7] established the following Ky Fan minimax inequalities for vector-valued mappings:

$$
\begin{aligned}
& \operatorname{Min}_{w} \bigcup_{x \in X_{0}} \operatorname{Max}_{w} f\left(x, X_{0}\right) \subset \operatorname{Max} \bigcup_{x \in X_{0}} f(x, x)-S, \\
& \operatorname{Max}_{w} \bigcup_{x \in X_{0}} f(x, x) \subset \operatorname{Min} \bigcup_{x \in X_{0}} \operatorname{Max}_{w} f\left(x, X_{0}\right)+S .
\end{aligned}
$$

Luo [8] also obtained some generalized Ky Fan minimax inequalities for vectorvalued mappings by applying the classical Browder fixed point theorem and the Kakutani-Fan-Glicksberg fixed point theorem.

There are also many articles to study the minimax theorems for vector-valued mappings. 
Nieuwenhuis [9] proved that

$$
\begin{aligned}
& \operatorname{Min} \bigcup_{y \in Y_{0}} \operatorname{Max}_{w} f\left(X_{0}, y\right) \subset \operatorname{Max} \bigcup_{x \in X_{0}} \operatorname{Min}_{w} f\left(x, Y_{0}\right)-S, \\
& \operatorname{Max} \bigcup_{x \in X_{0}} \operatorname{Min}_{w} f\left(x, Y_{0}\right) \subset \operatorname{Min} \bigcup_{y \in Y_{0}} \operatorname{Max}_{w} f\left(X_{0}, y\right)+S,
\end{aligned}
$$

where the vector-valued function is $f(x, y)=x+y$. Tanaka [10-12] obtained minimax theorems of the separated vector-valued function of the type $f(x, y)=u(x)+v(y)$ and investigated some existence results of cone saddle points for general vector-valued functions. Furthermore, by using the existence results of cone saddle points for vectorvalued mappings, he obtained the following result:

$$
\exists z_{1} \in \operatorname{Min} \bigcup_{y \in Y_{0}} \operatorname{Max}_{w} f\left(X_{0}, y\right) \text { and } \exists z_{2} \in \operatorname{Max} \bigcup_{x \in X_{0}} \operatorname{Min}_{w} f\left(x, Y_{0}\right)
$$

such that

$$
z_{1} \in z_{2}-S
$$

Shi and Ling [13] proved, respectively, a minimax theorem and a cone saddle point theorem for a class of vector-valued functions, which include the separated functions as its proper subset. Ferro $[14,15]$ studied minimax theorems for general vector-valued functions. Gong [16] obtained a strong minimax theorem and established an equivalent relationship between the strong minimax inequality and a strong cone saddle point theorem for vector-valued functions. Li et al. [17] investigated a minimax theorem and a saddle point theorem for vector-valued functions in the sense of lexicographic order, respectively.

To the best of authors' knowledge, there are few articles to investigate minimax problems for set-valued mappings. Li et al. [18] obtained some minimax inequalities for set-valued mappings by using a section theorem and a linear scalarization function. Li et al. [19] studied some generalized minimax theorems for set-valued mappings by using a nonlinear scalarization function. Zhang et al. [20] investigated some minimax problems for set-valued mappings by applying the Fan-Browder Fixed Point Theorem. Motivated by the study of $[7,10,13,18,20]$, we obtain two types of Ky Fan minimax inequalities for set-valued mappings.

The rest of the article is organized as follows. In Section 2, we introduce notations and preliminary results. In Section 3, we obtain two types of Ky Fan minimax inequalities for set-valued mappings. We also give some examples to illustrate our results.

\section{Preliminaries}

Let $X$ and $V$ be real Hausdorff topological vector spaces. Assume that $S$ is a pointed closed convex cone in $V$ with its interior int $S \neq \emptyset$. Some fundamental terminologies are presented as follows.

Definition 2.1 [21] Let $A \subset V$ be a nonempty subset.

(i) A point $z \in A$ is said to be a minimal point of $A$ iff $A \cap(z-S)=\{z\}$, and Min $A$ denotes the set of all minimal points of $A$.

(ii) A point $z \in A$ is said to be a weakly minimal point of $A$ iff $A \cap(z-\operatorname{int} S)=\varnothing$, and $\operatorname{Min}_{w} A$ denotes the set of all weakly minimal points of $A$. 
(iii) A point $z \in A$ is said to be a maximal point of $A$ iff $A \cap(z+S)=\{z\}$, and $\operatorname{Max} A$ denotes the set of all maximal points of $A$.

(iv) A point $z \in A$ is said to be a weakly maximal point of $A$ iff $A \cap(z+\operatorname{int} S)=\varnothing$, and $\operatorname{Max}_{w}$ denotes the set of all weakly maximal points of $A$.

It is easy to verity that

$\operatorname{Min} A \subset \operatorname{Min}_{w} A$ and $\operatorname{Max} A \subset \operatorname{Max}_{w} A$.

Definition 2.2 [22] Let $F: X \rightarrow 2^{V}$ be a set-valued mapping with nonempty values.

(i) $F$ is said to be upper semicontinuous (u.s.c.) at $x_{0} \in X$, iff for any neighborhood $N$ $\left(F\left(x_{0}\right)\right)$ of $F\left(x_{0}\right)$, there exists a neighborhood $N\left(x_{0}\right)$ of $x_{0}$ such that

$$
F(x) \subset N\left(F\left(x_{0}\right)\right), \quad \forall x \in N\left(x_{0}\right) .
$$

(ii) $F$ is said to be lower semicontinuous (l.s.c.) at $x_{0} \in X$, iff for any open neighborhood $N$ in $V$ satisfying $F\left(x_{0}\right) \cap N \neq \varnothing$, there exists a neighborhood $N\left(x_{0}\right)$ of $x_{0}$ such that

$$
F(x) \bigcap N \neq \emptyset, \quad \forall x \in N\left(x_{0}\right) .
$$

(iii) $F$ is said to be continuous at $x_{0} \in X$ iff $F$ is both u.s.c. and l.s.c. at $x_{0}$.

Remark 2.1 [22] The nonempty compact-valued mapping $F$ is said to be u.s.c. at $x_{0}$ $\in X_{0}$ if and only if for any net $\left\{x_{\alpha}\right\} \subset X$ with $x_{\alpha} \rightarrow x_{0}$ and for any $y_{\alpha} \in F\left(x_{\alpha}\right)$, there exist $y_{0} \in F\left(x_{0}\right)$ and a subnet $\left\{y_{\beta}\right\}$ of $\left\{y_{\alpha}\right\}$, such that $y_{\beta} \rightarrow y_{0}$.

Definition 2.3 Let $X_{0}$ be a nonempty convex subset of $X$, and let $F: X_{0} \rightarrow 2^{V}$ be a set-valued mapping with nonempty values.

(i) $F$ is said to be properly $S$-quasiconvex on $X_{0}$, iff for any $x_{1}, x_{2} \in X_{0}$ and $l \in[0,1]$, either

$$
F\left(x_{1}\right) \subset F\left(l x_{1}+(1-l) x_{2}\right)+S \text { or } F\left(x_{2}\right) \subset F\left(l x_{1}+(1-l) x_{2}\right)+S .
$$

$F$ is said to be properly $S$-quasiconcave on $X_{0}$, iff - $F$ is properly $S$-quasiconvex on $X_{0}$.

(ii) $F$ is said to be $S$-quasiconvex [23] on $X_{0}$, iff for any point $z \in V$, the level set

$$
\operatorname{Lev}_{F}(z):=\left\{x \in X_{0}: \exists t \in F(x) \text { s.t. } t \in z-S\right\}
$$

is convex. $F$ is said to be $S$-quasiconcave on $X_{0}$, iff - $F$ is $S$-quasiconvex on $X_{0}$.

Remark 2.2 If $F$ is a vector-valued mapping, then properly $S$-quasiconvex reduces to the ordinary properly $S$-quasiconvex in [14].

Lemma 2.1 Let $X_{0}$ be a compact subset of $X$. Suppose that $F: X_{0} \times X_{0} \rightarrow 2^{V}$ is a continuous set-valued mapping and for each $(x, y) \in X_{0} \times X_{0}, F(x, y)$ is a compact set. Then $\Gamma(y)=\operatorname{Min}_{w} \bigcap_{x \in X_{0}} F(x, y)$ and $\Phi(x)=\operatorname{Max}_{w} \bigcup_{y \in X_{0}} F(x, y)$ are u.s.c. and compactvalued on $X_{0}$, respectively.

Proof. It follows from Lemma 2.2 in [18] that $\Gamma$ and $\Phi$ are u.s.c. By the compactness of $X_{0}$ and the closeness of weakly minimal (maximal) point sets, $\Gamma$ and $\Phi$ are also compact-valued.

Lemma 2.2 [22] Let $X_{0}$ be a nonempty subset of $X$, and let $F: X_{0} \rightarrow 2^{V}$ be a setvalued mapping with nonempty values. If $X_{0}$ is compact and if $F$ is u.s.c. and compactvalued, then $F\left(X_{0}\right)=\bigcup_{x \in X_{0}} F(x)$ is compact. 
Lemma 2.3 [14] Let $A \subset V$ be a nonempty compact subset. Then (i) Min $A \neq \varnothing$; (ii) $A$ $\subset \operatorname{Min} A+S$; (iii) $A \subset \operatorname{Min}_{w} A+\operatorname{int} S \cup\left\{0_{V}\right\} ;($ iv) $\operatorname{Max} A \neq \varnothing ;(v) A \subset \operatorname{Max} A-S$; and (vi) $A \subset \operatorname{Max}_{w} A-\operatorname{int} S \cup\left\{0_{V}\right\}$.

Lemma 2.4 [24](Kakutani-Fan-Glicksberg fixed point theorem) Let $X_{0}$ be a nonempty compact convex subset of $X$. If $T: X_{0} \rightarrow 2^{X_{0}}$ is u.s.c, and for any $x \in X_{0}, T(x)$ is a nonempty, closed and convex set, then $T$ has a fixed point.

\section{Ky Fan minimax inequalities for set-valued mappings}

First, we prove the following interesting lemma.

Lemma 3.1 Let $X_{0}$ be a nonempty compact convex subset of $X$, and let $F: X_{0} \times X_{0} \rightarrow$ $2^{V}$ be a continuous set-valued mapping with nonempty compact values.

(i) If for each $x \in X_{0}, F(x, \cdot)$ is properly S-quasiconcave on $X_{0}$, then there exists $\bar{x} \in X_{0}$ such that

$$
F(\bar{x}, \bar{x}) \bigcap \operatorname{Max}_{w} \bigcup_{y \in X_{0}} F(\bar{x}, y) \neq \emptyset .
$$

(ii) If for each $y \in X_{0}, F(\cdot, y)$ is properly S-quasiconvex on $X_{0}$, then there exists $\bar{y} \in X_{0}$ such that

$$
F(\bar{y}, \bar{\gamma}) \bigcap \operatorname{Min}_{w} \bigcup_{x \in X_{0}} F(x, \bar{\gamma}) \neq \emptyset .
$$

Proof. (i) We define a multifunction $T: X_{0} \rightarrow 2^{X_{0}}$ by the formula

$$
T(x)=\left\{y \in X_{0}: F(x, y) \bigcap \operatorname{Max}_{w} \bigcup_{y \in X_{0}} F(x, y) \neq \emptyset\right\}, \quad \text { for } x \in X_{0} .
$$

First, we show that $T(x) \neq \varnothing$, for each $x \in X_{0}$. Since $F(x, \cdot)$ is u.s.c. with compact values and $X_{0}$ is compact, by Lemma 2.2, $\bigcup_{y \in X_{0}} F(x, y)$ is a compact set for each $x \in$ $X_{0}$. By Lemma 2.3, $\operatorname{Max}_{w} \bigcup_{y \in X_{0}} F(x, y) \neq \emptyset$. For each $x \in X_{0}$, let $z_{x} \in \operatorname{Max}_{w} \bigcup_{y \in X_{0}} F(x, y)$. Then, there exists $y_{x} \in X_{0}$ such that $z_{x} \in F\left(x, y_{x}\right)$. Namely,

$$
y_{x} \in T(x)=\left\{y \in X_{0}: F(x, y) \bigcap \operatorname{Max}_{w} \bigcup_{y \in X_{0}} F(x, y) \neq \emptyset\right\} .
$$

Hence, for each $x \in X_{0}, T(x) \neq \varnothing$.

Second, we show that $T(x)$ is a closed set, for each $x \in X_{0}$. Let a net $\left\{y_{\alpha}: \alpha \in I\right\} \subset T$ $(x)$, for each $x \in X_{0}$ and $y_{\alpha} \rightarrow y_{0}$. By the definition of $T$, there exists $\left\{z_{\alpha}\right\}$ such that $z_{\alpha}$ $\in F\left(x, y_{\alpha}\right)$ and $z_{\alpha} \in \operatorname{Max}_{w} \bigcup_{y \in X_{0}} F(x, y)$. Since $F(x, \cdot)$ is u.s.c. with nonempty compact values, by Remark 2.1 , there exist a subnet $\left\{z_{\beta}\right\}$ of $\left\{z_{\alpha}\right\}$ and $z_{0} \in F\left(x, y_{0}\right)$ satisfying $z_{\beta} \rightarrow$ $z_{0}$. By the closeness of the weakly maximal point set, $z_{0} \in \operatorname{Max}_{w} \bigcup_{y \in X_{0}} F(x, y)$. Thus, we have that

$$
y_{0} \in T(x)=\left\{y \in X_{0}: F(x, y) \bigcap \operatorname{Max}_{w} \bigcup_{y \in X_{0}} F(x, y) \neq \emptyset\right\}
$$


and hence for each $x \in X_{0}, T(x)$ is a closed set.

Now, we show that $T(x)$ is a convex set, for each $x \in X_{0}$. For each $x \in X_{0}$, let $y_{1}, y_{2}$ $\in T(x)$ and $l \in[0,1]$. Suppose that there exists $l_{0} \in[0,1]$ such that

$$
F\left(x, l_{0} y_{1}+\left(1-l_{0}\right) y_{2}\right) \bigcap \operatorname{Max}_{w} \bigcup_{y \in X_{0}} F(x, y)=\emptyset .
$$

Since $F\left(x, l_{0} y_{1}+\left(1-l_{0}\right) y_{2}\right) \subset F\left(x, X_{0}\right)$, by Lemma 2.3,

$$
F\left(x, l_{0} y_{1}+\left(1-l_{0}\right) y_{2}\right) \subset \operatorname{Max}_{w} \bigcup_{y \in X_{0}} F(x, y)-i n t S .
$$

Then, by assumptions and (1), we have that either

$$
F\left(x, y_{1}\right) \subset F\left(x, l_{0} y_{1}+\left(1-l_{0}\right) y_{2}\right)-S \subset \operatorname{Max}_{w} \bigcup_{y \in X_{0}} F(x, y)-\text { int } S
$$

or

$$
F\left(x, y_{2}\right) \subset F\left(x, l_{0} y_{1}+\left(1-l_{0}\right) y_{2}\right)-S \subset \operatorname{Max}_{w} \bigcup_{y \in X_{0}} F(x, y)-\text { int } S .
$$

Thus, we claim that either

$$
F\left(x, y_{1}\right) \bigcap \operatorname{Max}_{w} \bigcup_{y \in X_{0}} F(x, y)=\emptyset \quad \text { or } \quad F\left(x, y_{2}\right) \bigcap \operatorname{Max}_{w} \bigcup_{y \in X_{0}} F(x, y)=\emptyset .
$$

In fact, if (2) does not hold, i.e., there exist $z_{1}, z_{2} \in V$ such that

$$
z_{1} \in F\left(x, y_{1}\right) \bigcap \operatorname{Max}_{w} \bigcup_{y \in X_{0}} F(x, y) \text { and } z_{2} \in F\left(x, y_{2}\right) \bigcap \operatorname{Max}_{w} \bigcup_{y \in X_{0}} F(x, y) .
$$

Then, for $z_{1}, z_{2}$, there exist $z_{1}^{\prime}, z_{2}^{\prime} \in \operatorname{Max}_{w} \bigcup_{y \in X_{0}} F(x, y)$ such that either

$$
z_{1} \in z_{1}^{\prime}-\text { int } S \text { or } z_{2} \in z_{2}^{\prime}-\text { int } S .
$$

Clearly, this is a contradiction. Therefore, (2) holds, which also contradicts the assumption about $y_{1}$ and $y_{2}$. Hence, $T(x)$ is a convex set, for each $x \in X_{0}$.

Next, we show that $T$ is u.s.c. on $X_{0}$. Since $X_{0}$ is compact, we only need to show that $T$ is a closed map (see [22]). Let a net

$$
\left\{\left(x_{\alpha}, y_{\alpha}\right)\right\} \subset \operatorname{Graph} T:=\left\{(x, y) \in X_{0} \times X_{0}: F(x, y) \bigcap \operatorname{Max}_{w} \bigcup_{y \in X_{0}} F(x, y) \neq \emptyset\right\}
$$

and $\left(x_{\alpha}, y_{\alpha}\right) \rightarrow\left(x_{0} . y_{0}\right)$. By the definition of $T$, there exists $\left\{z_{\alpha}\right\}$ satisfying $z_{\alpha} \in F\left(x_{\alpha}\right.$, $\left.y_{\alpha}\right)$ and $z_{\alpha} \in \operatorname{Max}_{w} \bigcup_{y \in X_{0}} F\left(x_{\alpha}, y\right)$. By assumptions and Lemma 2.2, $\left\{z_{\alpha}\right\}$ must have a convergence subnet. For convenience, let the convergence subnet be itself. Since $F$ is u. s.c. with nonempty compact values, by Remark 2.1, there exist a subnet $\left\{z_{\beta}\right\}$ of $\left\{z_{\alpha}\right\}$ and $z_{0} \in F\left(x_{0}, y_{0}\right)$ satisfying

$$
z_{\beta} \rightarrow z_{0}
$$


By Lemma 2.1, $\operatorname{Max}_{w} \bigcup_{y \in X_{0}} F(\cdot, y)$ is u.s.c. and compact-valued. Then, by Remark 2.1 , there exist a subnet $\left\{z_{\gamma}\right\}$ of $\left\{z_{\alpha}\right\}$ and $z_{0}^{\prime} \in \operatorname{Max}_{w} \bigcup_{\gamma \in X_{0}} F\left(x_{0}, y\right)$ satisfying

$$
z_{\gamma} \rightarrow z_{0}^{\prime}
$$

Clearly, $z_{0}=z_{0}^{\prime}$. That is $\left(x_{0}, y_{0}\right) \in$ GraphT. Hence, $T$ is u.s.c. on $X_{0}$.

Therefore, by Lemma 2.4 , there exists $\bar{x} \in X_{0}$ such that $\bar{x} \in T(\bar{x})$, i.e.,

$$
F(\bar{x}, \bar{x}) \bigcap \operatorname{Max}_{w} \bigcup_{y \in X_{0}} F(\bar{x}, y) \neq \emptyset .
$$

(ii) We also define a multifunction $W: X_{0} \rightarrow 2^{X_{0}}$ by the formula

$$
W(y)=\left\{x \in X_{0}: F(y, y) \bigcap \operatorname{Min}_{w} \bigcup_{x \in X_{0}} F(x, y) \neq \emptyset\right\}, \quad \text { for } y \in X_{0}
$$

Similar to the above proof, we can prove that all conditions of Lemma 2.4 are satisfied. By Lemma 2.4, there exists $\bar{y} \in X_{0}$ such that $\bar{y} \in W(\bar{y})$, i.e.,

$$
F(\bar{y}, \bar{y}) \bigcap \operatorname{Min}_{w} \bigcup_{x \in X_{0}} F(x, \bar{y}) \neq \emptyset .
$$

$\square$

Remark 3.1 When $F$ is a real-valued function, Lemma 3.1 (i) reduces to Lemma 6 in [7].

Theorem 3.1 Let $X_{0}$ be a nonempty compact convex subset of X. Suppose that the following conditions are satisfied:

(i) $F: X_{0} \times X_{0} \rightarrow 2^{V}$ is a continuous set-valued mapping with nonempty compact values;

(ii) for each $x \in X_{0}, F(x, \cdot)$ is properly S-quasiconcave on $X_{0}$.

Then,

$$
\exists z_{1} \in \operatorname{Max} \bigcup_{x \in X_{0}} F(x, x) \text { and } \exists z_{2} \in \operatorname{Min} \bigcup_{x \in X_{0}} \operatorname{Max}_{w} F\left(x, X_{0}\right)
$$

such that

$$
z_{1} \in z_{2}+S
$$

Proof. By assumptions and Lemmas 2.1-2.3,

$$
\operatorname{Max} \bigcup_{x \in X_{0}} F(x, x) \neq \emptyset \text { and } \operatorname{Min} \bigcup_{x \in X_{0}} \operatorname{Max}_{w} F\left(x, X_{0}\right) \neq \emptyset \text {. }
$$

Then, by Lemma 3.1, there exists $\bar{x} \in X_{0}$ such that

$$
F(\bar{x}, \bar{x}) \bigcap \operatorname{Max}_{w} \bigcup_{y \in X_{0}} F(\bar{x}, y) \neq \emptyset .
$$

By Lemmas 2.1 and 2.2, $\bigcup_{x \in X_{0}} F(x, x)$ and $\bigcup_{x \in X_{0}} \operatorname{Max}_{w} F\left(x, X_{0}\right)$ are two compact sets. 
Thus, by Lemma 2.3, we have

$$
F(\bar{x}, \bar{x}) \subset \bigcup_{x \in X_{0}} F(x, x) \subset \operatorname{Max} \bigcup_{x \in X_{0}} F(x, x)-S
$$

and

$$
\operatorname{Max}_{w} \bigcup_{y \in X_{0}} F(\bar{x}, y) \subset \bigcup_{x \in X_{0}} \operatorname{Max}_{w} F\left(x, X_{0}\right) \subset \operatorname{Min} \bigcup_{x \in X_{0}} \operatorname{Max}_{w} F\left(x, X_{0}\right)+S .
$$

Namely, for every $u \in F(\bar{x}, \bar{x})$ and $v \in \operatorname{Max}_{w} \bigcup_{y \in X_{0}} F(\bar{x}, y)$, there exist $z_{1} \in \operatorname{Max} \bigcup_{x \in X_{0}} F(x, x)$ and $z_{2} \in \operatorname{Min} \bigcup_{x \in X_{0}} \operatorname{Max}_{w} F\left(x, X_{0}\right)$ such that

$$
u \in z_{1}-S \text { and } \quad v \in z_{2}+S \text {. }
$$

Particularly, taking $u=v$, we have $z_{1} \in z_{2}+S$. This completes the proof. $\square$

Corollary 3.1 Let $X_{0}$ be a nonempty compact convex subset of $X$. Suppose that the following conditions are satisfied:

(i) $f: X_{0} \times X_{0} \rightarrow V$ is a continuous vector-valued mapping;

(ii) for each $x \in X_{0}, f(x, \cdot)$ is properly S-quasiconcave on $X_{0}$.

Then,

$$
\exists z_{1} \in \operatorname{Max} \bigcup_{x \in X_{0}} f(x, x) \text { and } \exists z_{2} \in \operatorname{Min}_{w} \bigcup_{x \in X_{0}} \operatorname{Max}_{w} f\left(x, X_{0}\right)
$$

such that

$$
z_{1} \in z_{2}+S
$$

Proof. Since $\operatorname{Min} \bigcup_{x \in X_{0}} \operatorname{Max}_{w} f\left(x, X_{0}\right) \subset \operatorname{Min}_{w} \bigcup_{x \in X_{0}} \operatorname{Max}_{w} f\left(x, X_{0}\right)$, by the proof of Theorem 3.1, the conclusion follows readily. $\square$

Remark 3.2 Corollary 3.1 is different from Theorems 3 and 4 in [7] and Corollary 3.8 in [8]. The following example illustrates that when Theorem 3 in [7] and Corollary 3.8 in [8] are not applicable, Corollary 3.1 is applicable.

Example 3.1 Let $X=R, V=R^{2}, X_{0}=[0,1]$ and $S=\{(u, v) \mid u \geq 0, v \geq 0\}$. Let $f:[0,1]$ $\times[0,1] \rightarrow R^{2}$

$$
f(x, y)= \begin{cases}(x, 0), & x \leq y \\ (x, 2(x-y)), & x \geq y\end{cases}
$$

Obviously, $f$ is continuous and $f(x, \cdot)$ is properly $S$-quasiconcave for each $x \in X_{0}$. All conditions of Corollary 3.1 are satisfied. So, inclusion (4) holds. Indeed, by the definition of $f$, we have

$$
f(x, x)=(x, 0)
$$

and for each $x \in X_{0}$,

$$
\operatorname{Max}_{w} \bigcup_{y \in X_{0}} f(x, y)=\{(x, u) \mid 0 \leq u \leq 2 x\} .
$$


Then, by computing,

$$
\bigcup_{x \in X_{0}} f(x, x)=\{(u, 0) \mid 0 \leq u \leq 1\}
$$

and

$$
\bigcup_{x \in X_{0}} \operatorname{Max}_{w} f\left(x, X_{0}\right)=\{(u, v) \mid 0 \leq u \leq 1,0 \leq v \leq 2 u\} .
$$

Thus,

$$
\operatorname{Max} \bigcup_{x \in X_{0}} f(x, x)=\{(1,0)\}
$$

and

$$
\operatorname{Min}_{w} \bigcup_{x \in X_{0}} \operatorname{Max}_{w} f\left(x, X_{0}\right)=\{(u, 0) \mid 0 \leq u \leq 1\} .
$$

Taking $(0,0) \in \operatorname{Min}_{w} \bigcup_{x \in X_{0}} \operatorname{Max}_{w} f\left(x, X_{0}\right)$,

$$
(1,0) \in(0,0)+S
$$

However, taking $x_{0} \neq 1$, we have

$$
\operatorname{Min}_{w} \bigcup_{x \in X_{0}} \operatorname{Max}_{w} f\left(x, X_{0}\right) \not \subset \operatorname{Max}_{w} \bigcup_{y \in X_{0}} f\left(x_{0}, y\right)-S=f\left(x_{0}, X_{0}\right)-S .
$$

Namely, the condition (iii) of Theorem 3 in [7] and the condition (ii) of Corollary 3.8 in [8] do not hold. So, Theorem 3 in [7] and Corollary 3.8 in [8] are not applicable.

Theorem 3.2 Let $X_{0}$ be a nonempty compact convex subset of X. Suppose that the following conditions are satisfied:

(i) $F: X_{0} \times X_{0} \rightarrow 2^{V}$ is a continuous set-valued mapping with nonempty compact values;

(ii) for each $y \in X_{0}, F(\cdot, y)$ is properly S-quasiconvex on $X_{0}$.

Then,

$$
\exists z_{1} \in \operatorname{Min} \bigcup_{x \in X_{0}} F(x, x) \text { and } \exists z_{2} \in \operatorname{Max} \bigcup_{y \in X_{0}} \operatorname{Min}_{w} F\left(X_{0}, y\right)
$$

such that

$$
z_{1} \in z_{2}-S \text {. }
$$

Proof. By assumptions and Lemmas 2.1-2.3,

$$
\operatorname{Min} \bigcup_{x \in X_{0}} F(x, x) \neq \emptyset \text { and } \operatorname{Max} \bigcup_{y \in X_{0}} \operatorname{Min}_{w} F\left(X_{0}, y\right) \neq \emptyset
$$

Then, by Lemma 3.1, there exists $\bar{y} \in X_{0}$ such that

$$
F(\bar{y}, \bar{y}) \bigcap \operatorname{Min}_{w} \bigcup_{x \in X_{0}} F(x, \bar{y}) \neq \emptyset .
$$


By Lemmas 2.1 and 2.2, $\bigcup_{x \in X_{0}} F(x, x)$ and $\bigcup_{y \in X_{0}} \operatorname{Min}_{w} F\left(X_{0}, y\right)$ are two compact sets. Thus, by Lemma 2.3, we have

$$
F(\bar{y}, \bar{y}) \subset \bigcup_{x \in X_{0}} F(x, x) \subset \operatorname{Min} \bigcup_{x \in X_{0}} F(x, x)+S
$$

and

$$
\operatorname{Min}_{w} \bigcup_{x \in X_{0}} F(x, \bar{y}) \subset \bigcup_{y \in X_{0}} \operatorname{Min}_{w} F\left(X_{0}, y\right) \subset \operatorname{Max} \bigcup_{y \in X_{0}} \operatorname{Min}_{w} F\left(X_{0}, y\right)-S .
$$

Namely, for every $u \in F(\bar{y}, \bar{y})$ and $v \in \operatorname{Min}_{w} \bigcup_{x \in X_{0}} F(x, \bar{y})$, there exist $z_{1} \in \operatorname{Min} \bigcup_{x \in X_{0}} F(x, x)$ and $z_{2} \in \operatorname{Max} \bigcup_{y \in X_{0}} \operatorname{Min}_{w} F\left(X_{0}, y\right)$ such that

$$
u \in z_{1}+S \text { and } v \in z_{2}-S \text {. }
$$

Particularly, taking $u=v$, we have $z_{1} \in z_{2}-S$. This completes the proof. $\square$

Theorem 3.3 Let $X_{0}$ be compact convex subset of X. Suppose that the following conditions are satisfied:

(i) $F: X_{0} \times X_{0} \rightarrow 2^{V}$ is a continuous set-valued mapping with nonempty compact values;

(ii) for each $x \in X_{0}$, and any $z \in \operatorname{Min} \bigcup_{x \in X_{0}} \operatorname{Max}_{w} F\left(x, X_{0}\right)$, the level set

$$
\operatorname{Lev}_{F}(z)=\left\{y \in X_{0}: \exists t \in F(x, y) \text { s.t. } t \in z+S\right\}
$$

is convex.

(iii) for any $x \in X_{0}$,

$$
\operatorname{Min} \bigcup_{x \in X_{0}} \operatorname{Max}_{w} F\left(x, X_{0}\right) \subset F\left(x, X_{0}\right)-S
$$

Then,

$$
\operatorname{Min} \bigcup_{x \in X_{0}} \operatorname{Max}_{w} F\left(x, X_{0}\right) \subset \operatorname{Max} \bigcup_{x \in X_{0}} F(x, x)-S .
$$

Proof. By assumptions and Lemmas 2.1-2.3,

$$
\operatorname{Min} \bigcup_{x \in X_{0}} \operatorname{Max}_{w} F\left(x, X_{0}\right) \neq \emptyset .
$$

Let $\beta \in \operatorname{Min}_{x \in X_{0}} \operatorname{Max}_{w} F\left(x, X_{0}\right)$. We define a multifunction $W: X_{0} \rightarrow 2^{X_{0}}$ by the formula

$$
W(x)=\left\{y \in X_{0}: F(x, y) \bigcap(\beta+S) \neq \emptyset\right\}, \quad \text { for } x \in X_{0} .
$$

Obviously, by the conditions (ii) and (iii), we have that $W(x)$ is a nonempty convex set, for all $x \in X_{0}$.

Now, we show that $W(x)$ is a closed set, for any $x \in X_{0}$. Let a net $\left\{y_{\alpha}: \alpha \in I\right\} \subset W$ $(x)$, for each $x \in X_{0}$ and $y_{\alpha} \rightarrow y_{0}$. By the definition of $W$, there exists $\left\{z_{\alpha}\right\}$ such that $z_{\alpha}$ $\in F\left(x, y_{\alpha}\right)$ and $z_{\alpha} \in \beta+S$. Since $F(x, \cdot)$ is u.s.c. with compact values, by Remark 2.1, 
there exist a subnet $\left\{z_{\beta}\right\}$ of $\left\{z_{\alpha}\right\}$ and $z_{0} \in F\left(x, y_{0}\right)$ satisfying $z_{\beta} \rightarrow z_{0}$. By the closeness of $S, z_{0} \in \beta+S$. Thus, we have

$$
y_{0} \in W(x)=\left\{y \in X_{0}: F(x, y) \bigcap(\beta+S) \neq \emptyset\right\}
$$

and hence for each $x \in X_{0}, W(x)$ is a closed set.

Next, we show that $W$ is upper semicontinuous on $X_{0}$. Since $X_{0}$ is compact, we only need to show $W$ is a closed map (see [22]). Let a net

$$
\left\{\left(x_{\alpha}, y_{\alpha}\right)\right\} \subset \text { Graph } W:=\left\{(x, y) \in X_{0} \times X_{0}: F(x, y) \bigcap(\beta+S) \neq \emptyset\right\}
$$

and $\left(x_{\alpha}, y_{\alpha}\right) \rightarrow\left(x_{0} \cdot y_{0}\right)$. By the definition of $W$, there exists $\left\{z_{\alpha}\right\}$ satisfying $z_{\alpha} \in F\left(x_{\alpha}\right.$, $\left.y_{\alpha}\right)$ and $z_{\alpha} \in \beta+S$. Since $F$ is u.s.c. with compact values, by Remark 2.1, there exist a subnet $\left\{z_{\gamma}\right\}$ of $\left\{z_{\alpha}\right\}$ and $z_{0} \in F\left(x_{0}, y_{0}\right)$ satisfying $z_{\gamma} \rightarrow z_{0}$. By the closeness of $S, z_{0} \in \beta+$ $S$. That is $\left(x_{0}, y_{0}\right) \in$ Graph $W$. Namely, $W$ is upper semicontinuous on $X_{0}$.

Therefore, by Lemma 2.4, there exists $\bar{x} \in X_{0}$ such that $\bar{x} \in W(\bar{x})$, i.e.,

$$
F(\bar{x}, \bar{x}) \bigcap(\beta+S) \neq \emptyset .
$$

By (7) and Lemma 2.3, we have

$$
\beta \in F(\bar{x}, \bar{x})-S \subset \bigcup_{x \in X_{0}} F(x, x)-S \subset \operatorname{Max} \bigcup_{x \in X_{0}} F(x, x)-S .
$$

Hence, inclusion (6) holds. This completes the proof. $\square$

Remark 3.3 (i) The condition (ii) of Theorem 3.3 can be replaced by "for any $x \in$ $X_{0}, F(x, \cdot)$ is $S$-quasiconcave on $X_{0}$ ".

(ii) If $F$ is a scalar set-valued mapping, the condition (iii) of Theorem 3.3 always holds.

(iii) When $F$ is a vector-valued mapping, Theorem 3.3 reduces to corresponding ones in $[7,8]$.

Theorem 3.4 Let $X_{0}$ be a compact convex subset of $X$. Suppose that the following conditions are satisfied:

(i) $F: X_{0} \times X_{0} \rightarrow 2^{V}$ is a continuous set-valued mapping with nonempty compact values;

(ii) for each $y \in X_{0}$, and any $z \in \operatorname{Max} \bigcup_{y \in X_{0}} \operatorname{Min}_{w} F\left(X_{0}, y\right)$, the level set

$$
\operatorname{Lev}_{F}(z)=\left\{x \in X_{0}: \exists t \in F(x, y) \text { s.t. } t \in z-S\right\}
$$

is convex.

(iii) for any $y \in X_{0}$,

$$
\operatorname{Max} \bigcup_{y \in X_{0}} \operatorname{Min}_{w} F\left(X_{0}, y\right) \subset F\left(X_{0}, y\right)+S .
$$

Then,

$$
\operatorname{Max} \bigcup_{y \in X_{0}} \operatorname{Min}_{w} F\left(X_{0}, y\right) \subset \operatorname{Min} \bigcup_{x \in X_{0}} F(x, x)+S .
$$


Proof. By assumptions and Lemmas 2.1-2.3,

$$
\operatorname{Max} \bigcup_{\gamma \in X_{0}} \operatorname{Min}_{w} F\left(X_{0}, y\right) \neq \emptyset
$$

Let $\gamma \in \operatorname{Max} \bigcup_{\gamma \in X_{0}} \operatorname{Min}_{w} F\left(X_{0}, y\right)$. We define a multifunction $K: X_{0} \rightarrow 2^{X_{0}}$ by the formula

$$
K(\gamma)=\left\{x \in X_{0}: F(x, y) \bigcap(\gamma-S) \neq \emptyset\right\}, \quad \text { for } y \in X_{0} .
$$

From the proof process of Theorem 3.3, inclusion (8) holds. This completes the proof. $\square$

Remark 3.4 (i) The condition (ii) of Theorem 3.4 can be replaced by "for any $y \in$ $X_{0}, F(\cdot, y)$ is $S$-quasiconvex on $X_{0}$ “.

(ii) If $F$ is a scalar set-valued mapping, the condition (iii) of Theorem 3.4 always holds.

Next, we give an example for explaining Theorem 3.4.

Example 3.2 Let $X=R, V=R^{2}, X_{0}=[0,1] \subset X, S=\{(u, v) \mid u \geq 0, v \geq 0\}$, and $M=$ $\{(u, v) \mid 0 \leq u \leq 1,0 \leq v \leq 1\}$. Let $f:[0,1] \times[0,1] \rightarrow R^{2}$ and $F:[0,1] \times[0,1] \rightarrow 2^{R^{2}}$,

$$
f(x, y)=x(1, y), \quad \text { for }(x, y) \in[0,1] \times[0,1]
$$

and

$$
F(x, y)=f(x, y)+M
$$

Obviously, $F$ is continuous with nonempty compact values and $F(\cdot, y)$ is $S$-quasiconvex for every $y \in X_{0}$. By the definition of $F$,

$$
\bigcup_{u \in X_{0}} \operatorname{Min}_{w} F\left(X_{0}, y\right)=\{(u, 0) \mid 0 \leq u \leq 2\} \bigcup\{(0, v) \mid 0 \leq v \leq 1\}
$$

and for each $y \in X_{0}$,

$$
F\left(X_{0}, y\right)=\{(u, y u) \mid u \in[0,1]\}+M
$$

Moreover, by computing,

$$
\operatorname{Max} \bigcup_{\gamma \in X_{0}} \operatorname{Min}_{w} F\left(X_{0}, y\right)=\{(0,1)\} \bigcup\{(2,0)\} .
$$

Then, for each $y \in X_{0}$,

$$
\operatorname{Max} \bigcup_{y \in X_{0}} \operatorname{Min}_{w} F\left(X_{0}, y\right) \subset F\left(X_{0}, y\right)+S,
$$

namely, the condition (iii) of Theorem 3.4 holds. Thus, all conditions of Theorem 3.4 are satisfied. So, inclusion (8) holds. Indeed, we have that

$$
f(x, x)=\left(x, x^{2}\right)
$$

and

$$
\bigcup_{x \in X_{0}} F(x, x)=\left\{(u, v) \mid 0 \leq u \leq 1,0 \leq v \leq 1+u^{2}\right\} \bigcup\left\{(u, v) \mid 1 \leq u \leq 2,(u-1)^{2} \leq v \leq 2\right\} .
$$


Then,

$$
\operatorname{Min} \bigcup_{x \in X_{0}} F(x, x)=\{(0,0)\}
$$

Hence, we have that

$$
\operatorname{Max} \bigcup_{y \in X_{0}} \operatorname{Min}_{w} F\left(X_{0}, y\right) \subset \operatorname{Min} \bigcup_{x \in X_{0}} F(x, x)+S .
$$

\section{Acknowledgements}

The authors would like to thank the anonymous referees for their valuable comments and suggestions, which helped to improve the article. This study was supported by the National Natural Science Foundation of China No. 11171362.

\section{Author details}

${ }_{1}^{1}$ College of Mathematics and Statistics, Chongqing University, Chongqing 401331, China ${ }^{2}$ Mathematical Sciences Research Institute in Chongqing, Chongqing 401331, China

\section{Authors' contributions}

All authors contributed equally and significantly in writing this article. All authors read and approved the final manuscript.

\section{Competing interests}

The authors declare that they have no competing interests.

\section{Received: 21 June 2011 Accepted: 19 April 2012 Published: 19 April 2012}

\section{References}

1. Fan, K: A generalization of Tychondff's fixed point theorem. Math Ann. 142, 305-310 (1961)

2. Fan, K: A minimax inequality and applications, Inequalities III. pp. 103-111. Academic Press, New York and London (1972)

3. Ha, CW: On a minimax inequality of Ky Fan. Proc Am Math Soc. 99, 680-682 (1987)

4. Zhang, JH, Ma, R: Minimax inequalities of Ky Fan. Appl Math Lett. 6, 37-41 (1998)

5. Chen, GY: A generalized section theorem and a minimax inequality for a vector-valued mapping. Optimization. 22, 745-754 (1991)

6. Chang, SS, Lee, GM, Lee, BS: Minimax inequalities for vector-valued mappings on W-spaces. J Math Anal Appl. 198, 371-380 (1996)

7. Li, ZF, Wang, SY: A type of minimax inequality for vector-valued mappings. J Math Anal Appl. 227, 68-80 (1998)

8. Luo, XQ: On some generalized Ky Fan minimax inequalities. Fixed Point Theory Appl 2009, 9 (2009). Article ID 194671 doi:10.1155/2009/194671

9. Nieuwenhuis, JW: Some minimax theorems in vector-valued functions. J Optim Theory Appl. 40, 463-475 (1983)

10. Tanaka, T: Some minimax problems of vector-valued functions. J Optim Theory Appl. 59, 505-524 (1988)

11. Tanaka, T: Two types of minimax theorems for vector-valued functions. J Optim Theory Appl. 68, 321-334 (1991)

12. Tanaka, T: Existence theorems for cone saddle points of vector-valued functions in infinite-dimensional spaces. J Optim Theory Appl. 62, 127-138 (1989)

13. Shi, DS, Ling, C: Minimax theorems and cone saddle points of uniformly same-order vector-valued functions. J Optim Theory Appl. 84, 575-587 (1995)

14. Ferro, F: A minimax theorem for vector-valued functions. J Optim Theory Appl. 60, 19-31 (1989)

15. Ferro, F: A minimax theorem for vector-valued functions, Part 2. J Optim Theory Appl. 68, 35-48 (1991)

16. Gong, XH: The strong minimax theorem and strong saddle points of vector-valued functions. Nonlinear Anal. 68 , 2228-2241 (2008)

17. Li, XB, Li, SJ, Fang, ZM: A minimax theorem for vector valued functions in lexicographic order. Nonlinear Anal. 73 , $1101-1108(2010)$

18. Li, SJ, Chen, GY, Lee, GM: Minimax theorems for set-valued mappings. J Optim Theory Appl. 106, 183-200 (2000)

19. Li, SJ, Chen, GY, Teo, KL, Yang, XQ: Generalized minimax inequalities for set-valued mappings. J Math Anal Appl. 281, 707-723 (2003)

20. Zhang, Y, Li, SJ, Zhu, SK: Minimax problems for set-valued mappings. Numer Funct Aanl Optim. 33, 239-253 (2012)

21. Jahn, J: Vector Optimization: Theory, Applications, and Extensions. Springer, Berlin (2004)

22. Aubin, JP, Ekeland, I: Applied Nonlinear Analysis. John Wiley and Sons, New York (1984)

23. Luc, DT, Vargas, C: A saddle point theorem for set-valued maps. Nonlinear Anal. 18, 1-7 (1992)

24. Holmes, RB: Geometric Functional and it's Applications. Springer-Verlag, New York (1975)

doi:10.1186/1687-1812-2012-64

Cite this article as: Zhang and Li: Ky Fan minimax inequalities for set-valued mappings. Fixed Point Theory and Applications 2012 2012:64. 